\title{
Amelioration of Insulin, Leptin and Adiponectin Levels in Experimental Metabolic Syndrome Model by Some Drugs
}

\begin{abstract}
N. M. AL-RASHEED, H. M. ABDELKAREM ${ }^{1,2}$, L. M. FADDA, A. M. MOHAMED ${ }^{3,4}$, N. M. AL-RASHEED, Y. BASSIOUNI ${ }^{1}$, H. M. $^{2}$ ALI $^{5,6 *}$ AND A. H. GAFEER ${ }^{1}$

Department of Pharmacology, College of Pharmacy, King Saud University, Riyadh, KSA, ${ }^{1}$ Department Chemistry, Faculty of Science Aljouf University, Sakaka, ${ }^{2}$ Department of Nutrition, National Research Center, Dokki, Egypt, ${ }^{3}$ Biochemistry Department, Faculty of Science - Al Faisaliah, King Abdulaziz University, Jeddah, KSA, ${ }^{4}$ Therapeutic Chemistry Department, National Research Center, Dokki, Egypt, ${ }^{5}$ Department of Genetics and Cytology, National Research Centre, Dokki, Egypt, ${ }^{6}$ First Year Common Deanship, King Saud University, Riyadh-KSA
\end{abstract}

\section{Al-Rasheed, et al.: Amelioration of Insulin, Leptin and Adiponectin}

\begin{abstract}
A comparative study was planned to explore the modulating impact of metformin, Lipitor or orlistat against the metabolic syndrome components, including hyperglycemia and hyperlipidemia. Also, the effects of these drugs on leptin and adiponectin as biomarkers of metabolic syndrome in a rat model were investigated for the first time. The administration of these drugs each alone to metabolic syndrome induced rats markedly ameliorated the increase in blood glucose, normalized the serum insulin and the high-density lipoprotein cholesterol levels and reduced triglyceride, total cholesterol and low-density lipoprotein cholesterol. The alterations in serum leptin and adiponectin levels in metabolic syndrome induced rats were significantly modulated by these drugs. Metformin was the most effective drug in modulating most of the studied parameters.
\end{abstract}

Key words: Metabolic syndrome, adiponectin, leptin, orlistat, metformin

Metabolic syndrome (MS) is a cluster of metabolic abnormalities, including insulin resistance, hyperlipidemia, hypertension and abdominal adiposity, that directly raises the risk of the circulatory system abnormalities and type 2 diabetes mellitus (T2DM), causes mortality. This syndrome is associated with other comorbidities, including a prothrombotic state, proinflammatory state, reproductive disorders and non-alcoholic fatty liver disease ${ }^{[1]}$. The prevalence of the MS is increasing to epidemic proportions. Its prevalence varied from $8-43 \%$ in men and from $7-56 \%$ in women worldwide ${ }^{[2]}$. Adipose tissue hormones have essential influence in the prognosis of MS. Adipose tissue secretes polypeptide hormones, including adiponectin and leptin that contribute to the progress of obesity-associated illness including hypertension, atherosclerosis, $\mathrm{T}^{2} \mathrm{DM}^{[3]}$.

Adiponectin regulates carbohydrate and fat metabolism in insulin-sensitive tissues ${ }^{[4]}$. It increases fatty acid oxidation and glucose uptake in the muscle and reduces the synthesis of glucose in the liver. Its secretion is prohibited by pro-inflammatory cytokines, suggesting that inflammation might be an vital factor contributing

*Address for correspondence

E-mail: hsameh2312003@yahoo.com

November-December 2016 to hypoadiponectinemia in insulin resistant and obese states. The deficiency of adiponectin leads to insulin resistances progression, obesity, T2DM and atherosclerosis ${ }^{[5]}$. Adiponectin exerts profound anti-diabetic, antiatherogenic and antiinflammatory roles $^{[6]}$. In clinical studies, plasma adiponectin levels decrease as the obesity increase ${ }^{[7]}$, dyslipidemia ${ }^{[8]}$, diabetes $^{[9]}$ and cardiovascular disease ${ }^{[10]}$. A low plasma adiponectin level is observed in the $\mathrm{MS}^{[11]}$. Conversely, leptin is essential for the genesis of the MS and the cardiovascular disease ${ }^{[12]}$. Leptin is a multifunctional hormone takes part in controlling of energy exchange and body weight ${ }^{[13]}$. It is a key hormone in the regulation of body fat stores. This peptide controls energy metabolism at the level of hypothalamus by decreasing food intake and stimulating energy consumption ${ }^{[14]}$. High level of circulated leptin was reported in obese

This is an open access article distributed under the terms of the Creative Commons Attribution-NonCommercial-ShareAlike 3.0 License, which allows others to remix, tweak, and build upon the work non-commercially, as long as the author is credited and the new creations are licensed under the identical terms

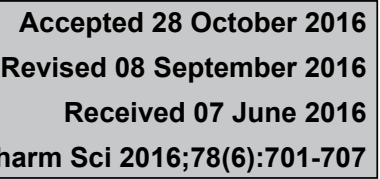

Indian J Pharm Sci 2016;78(6):701-707 
people due to leptin resistance ${ }^{[15]}$. Leptin resistance and hyperleptinemia take an important role in the development of insulin resistance ${ }^{[16]}$. Hyperlipidemia induces lipolysis by visceral fat, leads to accumulation of free fatty acids that inhibit the release of insulin from pancreatic $\beta$-cells ${ }^{[16]}$.

Metformin (Glucophage) is, 3-(diaminomethylidene)1,1-dimethylguanidine hydrochloride) an oral antidiabetic drug. It is the first line drug of choice for the treatment of T2DM, in particular, in overweight and obese people. Metformin works by suppressing glucose production in the liver, increasing peripheral glucose disposal, and reducing intestinal glucose absorption $^{[17]}$. It is the only antidiabetic drug that has been conclusively shown to prevent the cardiovascular complications during diabetes. It helps in reducing lowdensity lipoprotein (LDL) cholesterol and triglyceride levels and is not associated with weight gain ${ }^{[18]}$.

Atorvastatin (ATV), [(3R,5R)-7-[2-(4-Fluorophenyl)3-phenyl-4-(phenylcarbamoyl)-5-propan-2-ylpyrrol-1yl]-3,5-dihydroxyheptanoic acid], is a one of the drugs that are classified as statins marketed as Lipitor. This drug decreases the cholesterol level in the blood. It also stabilizes plaque and prevents strokes through its antiinflammatory effect and other mechanisms ${ }^{[19]}$. ATV works by inhibiting 3-hydroxy-3-methyl-glutarylcoenzyme a reductase (HMG-CoA), which is hepatic enzyme important for cholesterol production in the body. Statins are crucial drugs used to treat dyslipidemia and avoidance of circulatory systems disorders ${ }^{[19]}$.

Orlistat (Xenical), [(S)-((S)-1-((2S,3S)-3-hexyl4-oxooxetan-2-yl)tridecan-2-yl)-2-formamido-4methylpentanoate or tetrahydrolipstatin], is borrowed from lipstatin, a natural product of Streptomyces toxytricini. orlistat is a reversible inhibitor of gastric and pancreatic lipases ${ }^{[20]}$. It prohibits fat absorption from the human diet, by reduction of the intake of the calories. orlistat induces the reduction of the blood pressure and can prohibit the beginning of T2DM. It has been reported to reduce the incidence of diabetes by nearly $40 \%$ in obese people ${ }^{[21]}$.

Atherosclerosis is the lethal in hyperlipidemia people. This must be treated orally by drug that modulates the level of the glucose and rules out atherosclerosis progression. Unlikely, the choice of an effective medication is very limited ${ }^{[22]}$. From the above documented literature, adiponectin and leptin appear to have a bio-vital rule in MS progression. The currently available drugs have been developed for the treatment of one or more than one components of MS (e.g. antihyperglycemia, antihyperlipidemia) but the modulating effect of studied medications on adeponectin and leptin as biomarkers of MS is not explored.

The present study was planned to investigate the effect of metformin, Lipitor or orlistat on items of the MS such as hyperglycemia, hyperlipidemia and serum insulin in association with leptin and adiponectin levels in an experimental MS model. To the best of our knowledge, the effects of these drugs on leptin and adiponectin in MS induced in rats were investigated for the first time in the current study.

\section{MATERIALS AND METHODS}

A commercial formulation of fructose powder (Vivis, fructose sucrant poudre, France) was used. Metformin, Lipitor and orlistat were purchased from a local pharmacy. Kits for determination of serum triglycerides (TG), total cholesterol (TC), low-density lipoprotein (LDL-C), and high-density lipoprotein (HDL-C) were supplied by Randox Laboratories Ltd., UK. Insulin kit was obtained from American Laboratory Products Company, Windham. Leptin and adiponectin enzyme linked immunosorbent assay (ELISA) kits were obtained from Ani Biotech Oy, Orgenium Laboratories Division, and Vantaa, Finland. All chemicals used in the study were of high analytical grade supplied by Sigma and Merck companies.

\section{Experimental animals:}

Fifty male albino rats were obtained and treated in accordance with the Guiding Principles for the Care and Use of Laboratory Animals approved by Committee of King Saud University, College of Pharmacy (Riyadh, Saudi Arabia). The rats were housed in standard temperature and humidity with a 12:12-h light-dark cycle. The animals were given standard chow ad libitum for the duration of the study and allowed one week to adapt to the laboratory environment before drugs administration.

Animals were divided into five groups of 10 rats each and each animal was weighed at the beginning of the experiment. Control group: $\mathrm{G}_{1}$, rats were fed a modified diet containing 65\% corn starch (Cat no. d11708b, Research diet, USA), and allowed to drink tap water $a d$ libitum. $\mathrm{G}_{2}$ (MS induced group): rats were fed the same diet plus free access to $10 \%$ fructose $(\mathrm{w} / \mathrm{v})$ in drinking water for $8 \mathrm{w}$ to induce $\mathrm{MS}^{[23]}$. $\mathrm{G}_{3}$ (MS and metformin, $200 \mathrm{mg} / \mathrm{kg} / \mathrm{d})^{[24]}, \mathrm{G}_{4}$ (MS and Lipitor, $\left.75 \mathrm{mg} / \mathrm{kg} / \mathrm{d}\right)^{[25]}$ and $\mathrm{G}_{5}(\mathrm{MS} \text { and orlistat, } 200 \mathrm{mg} / \mathrm{kg} / \mathrm{d})^{[15]}$. 
All the drugs used were in tablet form, which were ground and suspended separately in $2 \%$ gum acacia and administered orally for $8 \mathrm{w}$ simultaneously with fructose ingestion. The body weight and food intake/ $\mathrm{kg}$ were measured once a week. The food intake was calculated per cage, using the following formula, food intake $=$ daily food intake $(\mathrm{g}) / \sum$ body weight of the rats in each cage $(\mathrm{g})$. The calculated daily food intake was approximately equivalent to $10 \mathrm{~g} / 100 \mathrm{~g}$.

\section{Biochemical assays:}

At the end of the experiment, rats were fasted overnight (12-14 h) and then weighed and anesthetized to collect the blood samples by heart puncture in dark tubes. The blood samples were then centrifuged at 3000 $\mathrm{g}$ for $10 \mathrm{~min}$ at $4^{\circ}$ for serum separation and stored at $-80^{\circ}$ until analyzed. Livers were weighed; serum glucose, total cholesterol, HDL-C, and TG levels were estimated colorimetrically. LDL-C was calculated using the Friedwald equation. Serum insulin, leptin, and adiponectin were measured using enzyme linked immunosorbent assay (ELISA). The reading was recorded using ELISA microplate reader (VERSA Max, Molecular Devices Corporation, MN, and USA).

\section{Statistical analysis:}

All values are expressed as mean \pm SE. Data were statistically analyzed using one way analysis of variance (ANOVA) for multiple group comparison, followed by Student's unpaired t-test for group comparison. Significance was set at $\mathrm{P} \leq 0.05$. Data were computed for statistical analysis by using SPSS software.

\section{RESULTS AND DISCUSSION}

The present results revealed that the body weight (BW) of rats that ingested high fructose was significantly higher than that of the untereated group. Treatment of rats with metformin, Lipitor or orlistat, significantly reduced the elevated body weight compared to the MS group. Nevertheless, there was a non-significant change in the weight of the liver between all groups was observed (Table 1).

Administration of metformin, Lipitor and/or orlistat effect on serum level of glucose and insulin in rats which ingested high fructose are presented in Table 2. Both glucose and insulin levels are increased significantly by high fructose comparing with normal rats. Treatment of rats with metformin, Lipitor, and orlistat, significantly reduced the elevated glucose level. The level of the insulin was significantly reduced in the metformin and orlistat treated groups compared with high fructose group.

Fig. 1 showed that ingestion of high fructose to rats caused a marked increase in serum triglycerides, total cholesterol, and LDL-C with a concomitant decrease in HDL-C compared with control normal rats. The alteration in lipid profile was significantly modulated by treating with metformin, Lipitor or orlistat.

The effect of the used drugs on serum leptin and adiponectin in rats ingested with high fructose is shown in fig. 2. From the fig. 2, it was observed that ingestion of high fructose to rats, markedly increased the level of serum leptin and decreased adiponectin vs. control animals. There was a significant increase in serum leptin level and a decrease in serum adiponectin levels. Administration of either metformin or orlistat significantly modulated the increase in leptin level and returned it nearly to a normal level. However, a nonsignificant change in leptin in rats treated with Lipitor compared with high fructose group. The three drugs effectively modulated the decrease in adiponectin level compared with high fructose group.

The worldwide pattern of disorder is shifting from infectious diseases to non-communicable diseases,

TABLE 1: EFFECT OF METFORMIN, LIPITOR AND OR ORLISTAT ON BODY AND LIVER WEIGHT IN CONTROL AND ALL TREATED GROUPS

\begin{tabular}{cccccc}
\hline Parameters & Control & MS & MS+metformin & MS+Lipitor & MS+Orlistat \\
\hline Body Weight BW $(\mathrm{g})$ & $218.9 \pm 6.04$ & $300.4 \pm 7.5^{* \mathrm{a}}$ & $238.3 \pm 8.5^{* \mathrm{~b}}$ & $258.2 \pm 6.3^{* \mathrm{~b}}$ & $253.2 \pm 8.3^{* \mathrm{~b}}$ \\
Liver Weight $(\mathrm{g}) / 100 \mathrm{~g} \mathrm{BW}$ & $3.3 \pm 0.7$ & $3.9 \pm 0.75$ & $3.46 \pm 0.6$ & $3.6 \pm 0.5$ & $3.55 \pm 0.8$ \\
\hline
\end{tabular}

Data are presented as mean \pm SD of 10 rats. Body weight was expressed in grams and liver weight was expressed as weight in grams per 100 $\mathrm{g}$ of body weight. ${ }^{\text {"a }} \mathrm{P} \leq 0.05$ compared with control group, ${ }^{\text {"b }} \mathrm{P} \leq 0.05$, compared with $M S$ group.

TABLE 2: SERUM GLUCOSE AND INSULIN LEVELS AFTER ADMINISTRATION OF METFORMIN, LIPITOR AND ORLISTAT IN FRUCTOSE FED RATS FOR 4-WEEK

\begin{tabular}{cccccc}
\hline Parameters & Control & MS & MS+metformin & MS+Lipitor & MS+Orlistat \\
\hline Glucose $(\mathrm{mg} / \mathrm{dl})$ & $80 \pm 9.6$ & $145.4 \pm 2.9^{\mathrm{a}}$ & $74.4 \pm 1.9^{*}$ & $81.6 \pm 4.7^{*}$ & $86.67 \pm 7.5^{*}$ \\
Insulin $(\mathrm{IU} / \mathrm{l})$ & $67.5 \pm 0.8$ & $74.8 \pm 1.4^{\mathrm{a}}$ & $68.01 \pm 0.95^{*}$ & $74.13 \pm 85^{\mathrm{a}}$ & $74.13 \pm 85^{\mathrm{a}^{*}}$ \\
\hline
\end{tabular}

Values are expressed as mean \pm SD. ${ }^{\mathrm{P}} \leq 0.05$ compared with control, * $\mathrm{P} \leq 0.05$, compared with $\mathrm{MS}$ group. 


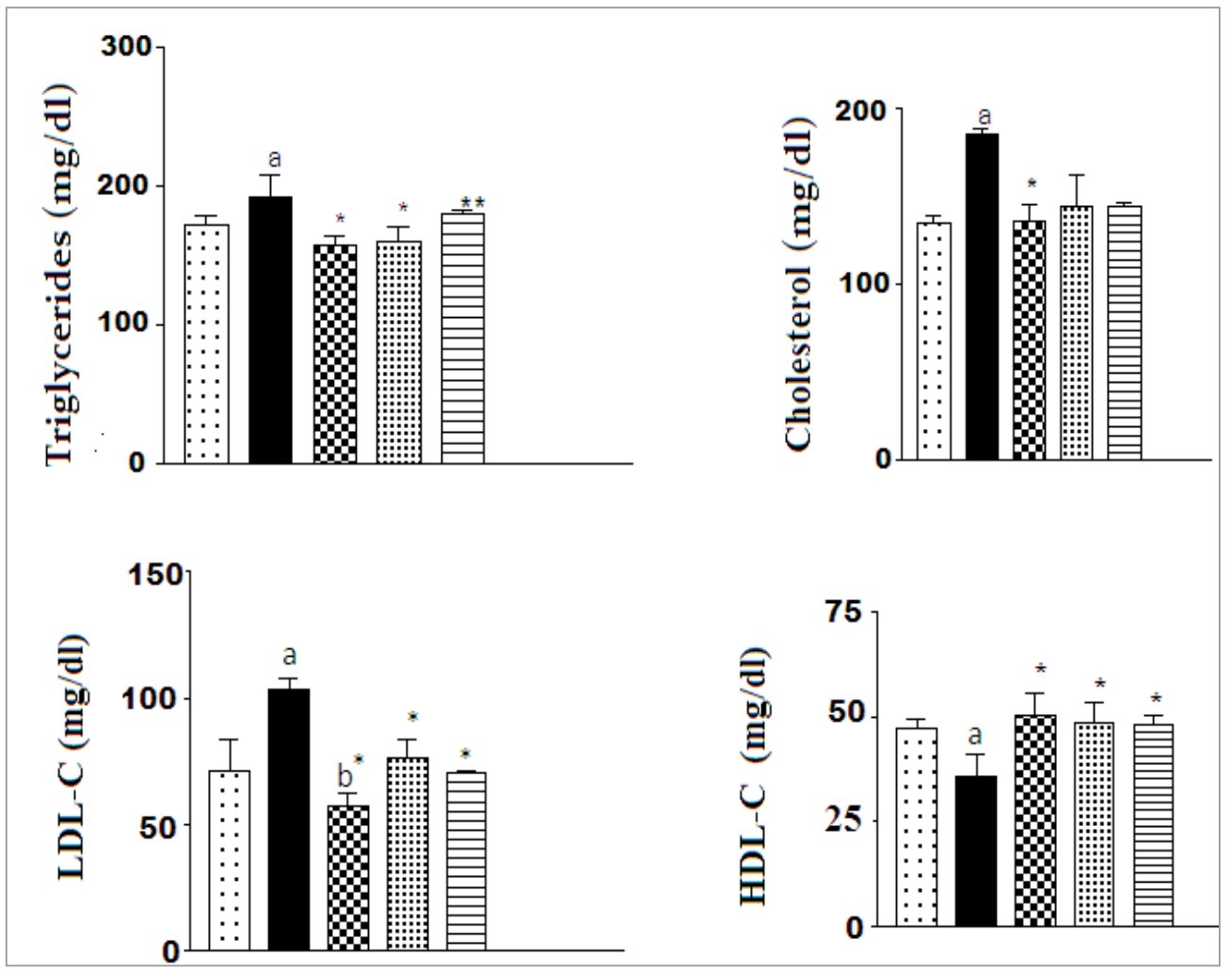

Fig. 1: Effect of metformin, Lipitor and orlistat on serum lipid profile of metabolic syndrome (MS) rats.

Values are expressed as mean \pm SD. ${ }^{a} \mathrm{P}<0.05$, compared with control $\mathrm{P}<0.05$ compared with MS group. ${ }^{\cdots} \mathrm{Control} ; \mathbf{M S}^{\square}$ metformin; Lipitor; 三orlistat.
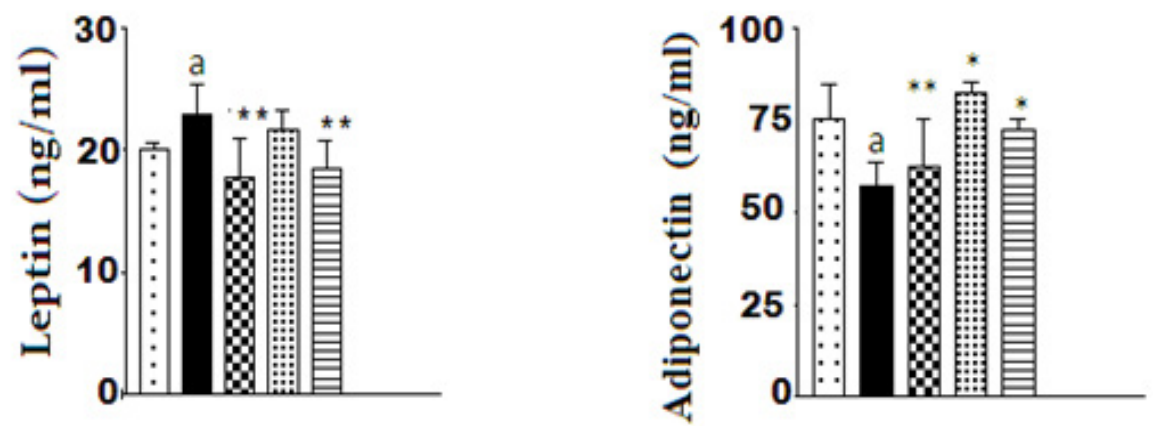

Fig. 2: Effect of metformin, Lipitor and orlistat on serum leptin and adiponectin of metabolic syndrome (MS) rats.

Values are expressed as mean \pm SD. ${ }^{a} \mathrm{P}<0.01$ compared with control, $\mathrm{P}<0.01$ compared with MS group. ${ }^{\cdots}$ control; ${ }_{\text {MS; }}$ metformin; Lipitor; $\equiv$ orlistat.

especially with heart diseases which are now being the chief causes of death globally. MS or "syndrome $X$ " is a collection of pathologies comprising insulin resistance, hyperinsulinemia, hypertriglyceridemia, accelerated atherosclerosis, and hypertension ${ }^{[1]}$. High dietary fructose has been correlated to the enhanced oxidative damage in rats and development of insulin resistance; $\beta$-cell dysfunction and defective glucose tolerance $^{[26]}$.

This is in harmony with the results of the present research, insulin, glucose, TG, total cholesterol and LDL-C serum levels likewise the body weight and the liver weight were significantly elevated, whereas likewise the body weight and the liver weight were significantly elevated. Administration of metformin, Lipitor and or orlistat downregulated the elevated glucose level compared with high fructose group. The inflated levels of (total cholesterol, LDL-C, and TG) were significantly suppressed by treating with metformin and Lipitor, respectively. However, orlistat administration induced no difference in serum TG. The level of HDL-C was significantly increased especially after administration of metformin comparing with high fructose group. 
The previous results were observed by some authors who clarified that metformin suppressed hyperglycemia by decreasing hepatic gluconeogenesis ${ }^{[27]}$. It also activates the enzyme AMP and activated protein kinase (AMPK), which plays a critical role in insulin signaling, energy balance in the body, and regulates the metabolism of glucose and fats ${ }^{[28]}$. It enhances insulin sensitivity, increases phosphorylating GLUT4 enhancer factor, so enhances peripheral glucose uptake which may be due to improved insulin binding to insulin receptors ${ }^{[28]}$. Also, the previous results are in accordance with those of Colhoun et al. ${ }^{[29]}$ Who clarified that atorvastatin was a selective, competitive inhibitor of HMG-CoA reductase used in patients with diabetes and hypercholesterolemia and has been found to be safe and effective. In an animal model of T2DM, atorvastatin exerts significant lipid and glucose lowering effects and decreased plasma insulin levels and insulin resistance index. Although the primary goal of atorvastatin is to improve blood lipid and lipoprotein characteristics, beneficial effects on energy metabolism and cardiovascular function such as improved endothelial function, reduced oxidative stress, decreased platelet adhesion and increased atherosclerotic plaque stability may help to explain at least part of the cardioprotective benefits of these agents ${ }^{[30]}$. The results of the present work were also supported by Harp ${ }^{[31]}$ who reported that the use of the pancreatic lipase inhibitor orlistat helps to lose weight due to decrease the absorption of dietary lipids. Treating of obese rats with orlistat reduce lipid profile including cholesterol, triglyceride and LDL.

Indeed, circadian oscillation of many hormones involved in metabolisms, such as corticosterone, insulin, glucagon, adiponectin, leptin and ghrelin, becomes disrupted in the progression of MS and obesity $^{[32]}$. Two major adipocytokines, leptin and adiponectin may perform vital rules in the managing of cardiovascular and metabolic homeostasis. Leptin acts directly on the hypothalamus, thereby regulating food intake and energy consumption ${ }^{[33]}$. Obesity decreases the adiponectin expression; hence it is expressed in the adipose tissue. Adiponectin influences insulin sensitivity, metabolism, inflammation and vascular function ${ }^{[34]}$.

Adiponectin decreases hepatic and muscular triglyceride content by reducing insulin resistance in obese mice, which leads to the enhancement of the hepatic insulin sensitivity and elevates peripheral glucose utilization in the liver and skeletal muscle ${ }^{[35]}$. Adiponectin also has beneficial effects on endothelial dysfunction by stimulating the production of nitric oxide. High circulating adiponectin levels are known to have many protective effects including antidiabetic, antiatherosclerotic and anticancer properties. Emerging evidence also stimulates nitric oxide production which improves endothelial injury. Its high levels protect against diabetic, atherosclerotic and cancer. Recent studies documented its capability to improve the lipid profile in blood by lipid-lowering therapies, and the modulation of adipose tissue adipokines synthesis and secretion. Ismail et al. ${ }^{[36]}$ reported that metformin improved insulin resistance in T2DM rats by normalizing serum lipid profiles in diabetic rats. Metformin upregulated adiponectin expression while leptin expression was decreased. Administration of ATV $(2.5 \mathrm{mg} / \mathrm{kg} / \mathrm{d})$ for $6 \mathrm{w}$ in hypercholesterolemic rabbits reduced serum leptin concentrations by $37.7 \%$. Moreover, its administration for $8 \mathrm{w}$ in human suffering of obesity and T2DM reduced plasma leptin concentrations by $40 \%$. It was reported ATV, pitavastatin and pravastatin elevated the serum adiponectin concentrations, and elevated plasma adiponectin levels in hypercholesterolemic human ${ }^{[37]}$.

Ozkan et al. ${ }^{[38]}$ reported that body mass index and the total blood cholesterol, LDL and triglyceride levels fell significantly after orlistat in the obese orlistattreated subjects. Also, leptin level was decreased in obese subjects treated with orlistat. The present work is parallel to those of the previous publications reported that high fructose diet induced an increase in serum leptin and a decrease in adiponectin levels in rats. Administration of either metformin or orlistat markedly modulated the increase in serum leptin level and returned it nearly to a normal level. However, no significant change of leptin level was observed upon Lipitor treatment compared with high fructose ingested group. The significant rise in adiponectin level was observed in animals treated with Lipitor, orlistat and metformin comparing with high fructose group.

In conclusion, the high fructose diet is a good design for detection of leptin and adiponectin in different animal models. Also, it can be concluded that the three drugs, metformin, Lipitor and/or orlistat, were effective in attenuating hyperglycemia and hyperlipidemia (MS) as well as in modulating the alteration in serum leptin and adiponectin levels induced in rats under the effect of high fructose ingestion. Metformin was the most effective drug in modulating the deviation in the in the studied biomarkers. It can be used either alone or in combination with Lipitor and or orlistat 
for best modulation effect. Based on this evidence it is recommended to use these drugs, particularly metformin in the protection and the treatment of metabolic syndrome.

\section{Acknowledgments:}

This research project was supported by a grant from Research Center of the Center for Female Scientific and Medical colleges", Deanship of Scientific Research, King Saud University.

\section{Conflicts of interest:}

The authors declared that there is no conflict of interest.

\section{Financial support and sponsorship:}

Author Laila M. Fadda has received research grants from the "Research Center of the Center for Female Scientific and Medical Colleges', Deanship of Scientific Research, King Saud University.

\section{REFERENCES}

1. Stump CS, Clark SE, Sowers JR. Oxidative stress in insulin resistant conditions: cardiovascular implications. Treat Endocrinol 2005;4:343-51.

2. Cameron AJ, Shaw JE, Zimmet PZ. The metabolic syndrome: prevalence in worldwide populations. Endocrinol Metab Clin North Am 2004;33:351-75.

3. Zahorska-Markiewicz B. Metabolic effects associated with adipose tissue distribution. Adv Med Sci 2006;51:111-14.

4. Diez JJ. The role of the novel adipocyte derived hormone adiponectin in human disease. Eur J Endocrinol 2003;148:293-300.

5. Lago F, Dieguez C, omez-Reino JG, Gualillo O. The emerging role of adipokines as mediators of inflammation and immune responses. Cytokine Growth Factor Rev 2007;18:313-25.

6. Kershaw EE, Flier JS. Adipose tissue as an endocrine organ. J Clin Endocrinol Metab 2004;89:2548-56.

7. Arita Y, Kihara S, Ouchi N, Takahashi M, Maeda K, Miyagawa $\mathrm{J}$, et al. Paradoxical decrease of an adipose specific protein, adiponectin, in obesity. Biochem Biophys Res Commun 1999;257:79-83.

8. Matsubara M, Maruoka S, Katayose S. Decreased plasma adiponectin concentrations in women with dyslipidemia. J Clin Endocrinol Metab 2002;86:2764-9.

9. Rothenbacher D, Brenner H, März W, Koenig W. Adiponectin, risk of coronary heart disease and correlations with cardiovascular risk markers. Eur Heart J 2005;26:1640-6.

10. Xydakis AM, Case CC, Jones PH, Hoogeveen RC, Liu MY, Smith EO, et al. Adiponectin, inflammation and the expression of the metabolic syndrome in obese individuals: the impact of rapid weight loss through caloric restriction. J Clin Endocrinol Metab 2004;89:2697-703.

11. Sader S, Nian M, Liu P. Leptin: a novel link between obesity, diabetes, cardiovascular risk, and ventricular hypertrophy. Circulation 2003;108:644-6.

12. Ahima RS. Leptin. Ann Rev Physiol 2000;62:413-37.
13. Steinberger J, Steffen L, Jacobs DR Jr, Moran A, Hong CP, Sinaiko AR. Relation of leptin to insulin resistance syndrome in children. Obes Res 2003;11:1124-30.

14. Koerner A, Kratzsch J, Kiess W. Adipocytokines: leptin the classical, resistin the controversial, adiponectin the promising, and more to come. Best Pract Res Clin Endocrinol Metab 2005;19:525-46.

15. Guerre-Millo M. Extending the glucose/fatty acid cycle: a glucose/adipose tissue cycle. Biochem Soc Trans 2003;31:1161-4.

16. El-Atat F, McFarlane SI, Sowers JR. Diabetes, hypertension, and cardiovascular derangements: pathophysiology and management. Curr Hypertens Rep 2004;6:215-23.

17. Selvin E, Bolen S, Yeh HC, Wiley C, Wilson LM, Marinopoulos SS, et al. Cardiovascular outcomes in trials of oral diabetes medications: a systematic review. Arch Inter Med 2008:168;2070-80.

18. Yu CM, Zhang Q, Lam L, Lin H, Kong SL, Chan W, et al. Comparison of intensive and low dose atorvastatin therapy in the reduction of carotid intimal medial thickness in patients with coronary heart disease. Heart 2007;93:933-99.

19. Tiss A, Lengsfeld H, Hadváry P, Cagna A, Verger R. Transfer of orlistat through oil water interfaces. Chem Physic Lipids 2002;119:41-9.

20. Padwal R, Li SK, Lau DC. Long term pharmacotherapy for obesity and overweight. Cochrane Database Sys Rev 2004;3:CD004094.

21. Pareek A, Yeole PG, Tenpe CR, Chandurkar N, Payghan R. Effect of atorvastatin and hydroxychloroquine combination on blood glucose in alloxan-induced diabetic rats. Indian $\mathrm{J}$ Pharmacol 2009;41:125-8.

22. Sánchez-Lozada LG, Tapia E, Jiménez A, Bautista P, Cristóbal $\mathrm{M}$, Nepomuceno $\mathrm{T}$, et al. Fructose induced metabolic syndrome is associated with glomerular hypertension and renal microvascular damage in rats. Am J Physiol Renal Physiol 2007;292:F423-9.

23. Smith DL Jr, Elam CF Jr, Mattison JA, Lane MA, Roth GS, Ingram DK, et al. Metformin supplementation and life span in Fischer-344 rats. J Gerontol A Biol Sci 2010;65:468-74.

24. Maurya DI, Belgamwar V, Tekade A. Microwave-induced solubility enhancement of poorly water soluble atorvastatin calcium. J Pharm Pharmacol 2010;62:599-1606.

25. Cruz-Hernandez C, Oliveira M, Pescia G, Moulin J, MassereyElmelegy I, Dionisi F, et al. Lipase inhibitor orlistat decreases incorporation of eicosapentaenoic and docosahexaenoic acids in rat tissues. Nutr Res 2010;30:134-40.

26. Bloch-Damti A, Bashan N. Proposed mechanisms for the induction of insulin resistance by oxidative stress. Antioxid Redox Signal 2005;7:1553-67.

27. Kirpichnikov D, McFarlane SI, Sowers JR. Metformin: an update. Ann Intern Med 2002;137:25-33.

28. Towler MC, Hardie DG. AMP-activated protein kinase in metabolic control and insulin signaling. Circ Res 2007;100:328-41.

29. Colhoun HM, Betteridge DJ, Durrington PN, Hitman GA, Neil HA, Livingstone SJ, et al. Primary prevention of cardiovascular disease with atorvastatin in type 2 diabetes in the Collaborative Atorvastatin Diabetes Study (CARDS): multicentre randomized placebo-controlled trial. Lancet 2004;364:685-96. 
30. Wanders D, Plaisance EP, Judd RL. Pharmacological effects of lipid-lowering drugs on circulating adipokines. World $\mathrm{J}$ Diabetes 2010;15:116-28.

31. Harp JB. An assessment of the efficacy and safety of orlistat for long-term management of obesity. J Nutr Biochem 1998;9:516-21.

32. Froy $\mathrm{O}$. The relationship between nutrition and circadian rhythms in mammals. Front Neuroendocrinol 2007;28:61-71.

33. Friedman JM, Halaas JL. Leptin and theregulation of body weight in mammals. Nature 1998;395:763-70.

34. Enriori PJ, Evans EA, Sinnayah P. Colwey MA. Leptin Resistance and Obesity. Obesity 2006;14:254S-8S.
35. Huypens P. Leptin controls adiponectin production in the hypothalamus. Med Hypotheses 2007;68:87-90.

36. Ismail TA, Soliman MM, Ismail SA. Adiponectin regulation in type 2 diabeticrats: Effect of insulin, metfomin and dexamethasone. J Pharmacol Toxicol 2013;8:197-208.

37. Gannagé-Yared MH, Azar RR, Amm-Azar M, Khalifé S, Germanos-Haddad M, Neemtallah R, et al. Pravastatin does not affect insulin sensitivity and adipocytokines levels in healthy nondiabetic patients. Metabolism 2005;54:947-51.

38. Ozkan Y, Aydin S, Donder E, Koca SS, Aydin S, Ozkan B, et al. Effect of orlistat on the total ghrelin and leptin levels in obese patients. J Physiol Biochem 2009;65:215-24. 